\title{
Craniotomy Flap Bone Cement Infections
}

Sarah F. Wesley ${ }^{1}$, Jacqueline M. Regan ${ }^{2}$, Joe C. Watson ${ }^{3}$

1. Mount Sinai Beth Israel Medical Center 2. University of Michigan, University of Michigan 3. Department of Neurosurgery, Virginia Commonwealth University

$\square$ Corresponding author: Jacqueline M. Regan, jackre@umich.edu Disclosures can be found in Additional Information at the end of the article

\section{Abstract \\ Introduction}

Kryptonite ${ }^{\circledR}$ is calcium triglyceride bone cement designed for cranial use. Despite potential benefits, Kryptonite ${ }^{\circledR}$ lacks safety data addressing its possible association with post-surgical wound infections. Upon noting an acute rise in the number of post-craniotomy infections while using Kryptonite Bone Cement ${ }^{\circledR}$, we investigated the hypothesis that using Kryptonite ${ }^{\circledR}$ increases the risk of post-craniotomy wound infection.

\section{Methods}

With institutional review board approval, a retrospective cohort study was performed using data on all craniotomies performed at single hospital in the United States over 32 months. Incidence of infection in both exposed group (Kryptonite ${ }^{\circledR}$ ) and unexposed group (Synthes@ plates and screws) were culled from hospital medical records for statistical analysis. Surgical and patient details, along with involved microorganisms, were also noted.

\section{Results}

Post-craniotomy infections in exposed and unexposed groups were $17.8 \%$ and $0.42 \%$, respectively. The relative risk of infection in the exposed group using Kryptonite ${ }^{\circledR}$ versus the unexposed group using plates and screws was 42.1 (95\% CI $=5.10-347.95, \mathrm{P}=0.0001)$. The attributable risk in the exposed was 98 percent $(95 \% \mathrm{CI}=0.804-0.997, \mathrm{P}=0.0001)$. Infected cases had each been exposed to a different batch of Kryptonite ${ }^{\circledR}$, and there were a wide variety of microorganisms involved.

\section{Conclusions}

Kryptonite ${ }^{\circledR}$ was associated with an increased risk of post-surgical cranial infections in this single center retrospective review relative to plates and screws. Larger multicenter studies are needed to evaluate infection rates when using bone-cement cranial fixation products. Until such studies are completed, it is recommended that these products be used with caution, particularly in immunocompromised patients and in those with redo procedures on prior infected sites. 
Reapproximation of a cranial flap with a bone glue has theoretical appeal for surgeons and patients as it could eliminate the need for metallic fixation and improve cosmetic contours. Kryptonite ${ }^{\circledR}$ is a non-toxic calcium triglyceride bone cement manufactured by the Doctors Research Group, Inc. (Southbury, CT). While it has been used in Europe for many years, Kryptonite ${ }^{\circledR}$ was only approved by the U.S. Food and Drug Administration (FDA) in 2009 [1] and has since been used in many institutions for cranial bone flap replacement as an alternative to traditional plates and screws. The manufacturer has claimed that Kryptonite ${ }^{\circledR}$ is nongenotoxic, non-hemolytic, and non-mutagenic, unlike older bone cements that contained polymethyl methacrylate (PMMA), a compound linked to tissue necrosis and peri-operative environmental hazard from toxic fumes. Moreover, unlike plates and screws, Kryptonite ${ }^{\circledR}$ polymerizes in stages, thus going from a liquid to a malleable putty form as it hardens for easier bone-to-bone adhesion [2]. Despite these benefits, Kryptonite ${ }^{\circledR}$ lacks safety data regarding its postoperative wound hygiene. There are no studies addressing risk of infection with older PMMA-based bone cement, nor have there been previous studies revealing risk of infection with Kryptonite ${ }^{\circledR}$. However, upon noting a spike in post-craniotomy infections after switching from traditional plates and screws to Kryptonite ${ }^{\circledR}$, a retrospective cohort study was conducted at one institution to investigate any potential association between exposure to Kryptonite ${ }^{\circledR}$ and an outcome of wound infection. Subsequently, the hypothesis was formulated that postcraniotomy wound infection rates were higher in the exposed group using Kryptonite ${ }^{\circledR}$ than in the unexposed group with Synthes@ plates and screws.

\section{Materials And Methods}

A retrospective cohort study investigating the outcome of post-surgical infection was performed, with Inova Human Research Protection Program (formerly IRB) approval (protocol \#12-1063), using data from 32 months of hospital medical records on all craniotomies performed at a single hospital in Virginia, USA, between January 2009 and August 2011. Consent was formally obtained or waived for all subjects present within this study. The incidence of infection from this period was determined from hospital epidemiological records confirmed with positive cultures. Where available, types of involved microorganisms and locations of infection were also recorded.

A comparison between relative risk of post-surgical infection in the group exposed to Kryptonite ${ }^{\circledR}(n=28)$ versus in the unexposed group that used Synthes $\odot$ plates and screws $(n=$ 180) was analyzed for attributable risk and statistical significance.

Statistical analysis was performing using Stata statistical software on the dataset (StataCorp LP, College Station, TX). A 2x2 table of cases, non-cases, exposed, and unexposed was constructed with this software to determine risk difference, risk ratio, attributable fraction in exposed, and attributable fraction in the general population. Using 95 percent confidence intervals with a Fisher's two-sided test for statistical significance in a smaller sample size, the software was used to calculate statistical significance in the form of a $P$-value.

Inclusion criteria for the study were all patients who underwent a craniotomy from January 2009 to August $2011(n=208)$. This sample included two years of data for patients in the unexposed group on whom Synthes@ plates and screws were used to seal the bone flap of their craniotomies, as well as the group who received Kryptonite ${ }^{\circledR}$.

The study controlled for possible confounding variables. One patient in the infection group had had prior radiation. No patients had diabetes. All surgeries were performed by the same two neurosurgeons in the same two operating rooms at the same

hospital. All patients in both groups received 1-gram preoperative cefazolin intravenously prior to skin incision per our protocol and per our standard "Time Out" procedure. None of the infected patients received any other antibiotics preop, such as Vancomycin. There were several 


\section{Cureus}

patients in the uninfected craniotomy group (7\%) who received Vancomycin. Surgical technique involved a standard free flap craniotomy in all cases with application of the cement to both the flap and cranial edges, followed by replacement into anatomical position, and a waiting period of seven minutes prior to skin closure to allow for curing to the cement and cement expansion (Figure 1). No patient received postoperative prophylactic antibiotics in any group. All patients went directly to the post-anaesthesia care unit and were then transferred to the neuroscience intensive care unit. No patients had other postoperative systemic infections, such as pneumonia or pyelonephritis.

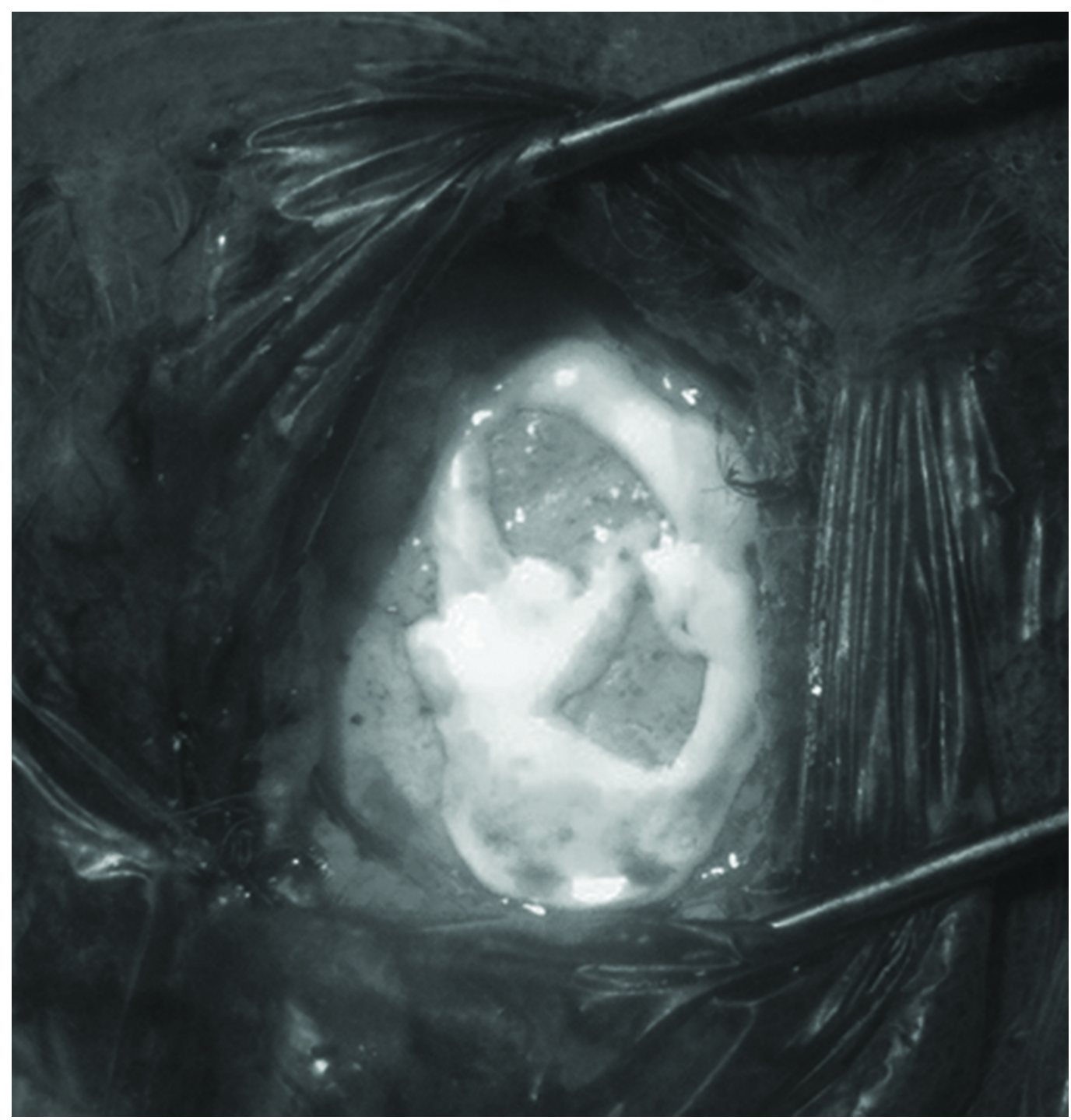

FIGURE 1: Intraoperative photograph of a craniotomy flap that has been fixated with Kryptonite ${ }^{\circledR}$ bone cement.

Exclusion criteria included patients with pre-existing scalp or skin infections, or any deep cranial infections.

\section{Results}

During the period of January 2009 through January 2011, a total of 180 craniotomies were performed by neurosurgeons who used traditional plates and screws as the method of skull 


\section{Cureus}

closure. There was one single postoperative infection during this period. The surgical wound culture grew Staphylococcus aureus for this case, which was subsequently treated with incision and drainage of the skin infection. No patients were excluded on the grounds of pre-surgical infection. In February 2011, the new product, Kryptonite ${ }^{\circledR}$, was used as a bone cement for 28 craniotomies, instead of plates and screws; however, after the department noted a sharp rise in the number of post-surgical infections, the use of the bone cement was terminated in May 2011. There were four postoperative infections in this three-month period alone. The following microorganisms were found on cultures, where information was available: Staphylococcus aureus, diptheroids, and Staphylococcus epidermidis (Table 1). A fifth patient presented 11 months postoperative with a draining wound and osteomyeltis from Proprionibacterium.

\begin{tabular}{|c|c|c|}
\hline Infected Cases & Type of Craniotomy & Type of Organism \\
\hline 1 & Right frontal, meningioma & Staphylococcus aureus \\
\hline 2 & Bi-frontal meningioma & Diptheroids and Staphylococcus epidermidis \\
\hline 3 & Left temporal glioblastoma & Staphylococcus epidermidis \\
\hline 4 & Right temporal Grade III astrocytoma & Microorganism type unavailable \\
\hline 5 & Right temporal lymphoma & Propionibacterium acnes \\
\hline
\end{tabular}

\section{TABLE 1: Known pathogens from infected cases}

All of these patients required removal of the craniotomy flap, prolonged intravenous antibiotics and late reconstructive cranioplasty. One patient suffered a venous infarct and frontal lobe stroke as a result of the infection (Figure 2). Of significance, operating room records indicate that the Kryptonite ${ }^{\circledR}$ had different lot numbers in all four infected cases. No patients had a known pre-surgical infection.

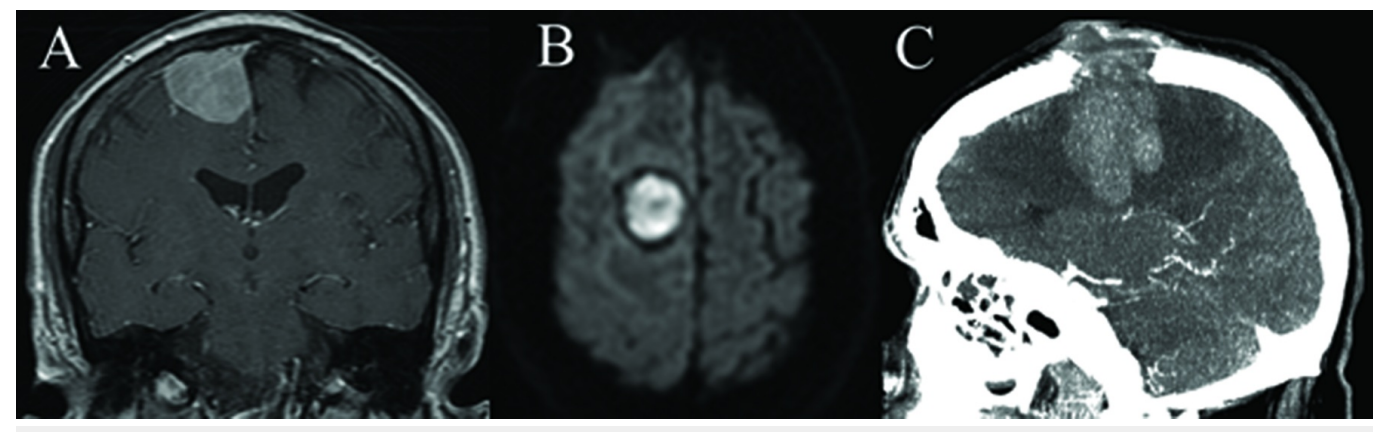

FIGURE 2:

Imaging from one of our Kryptonite $₫$ flap patients who become infected: A) preoperative coronal post-contrast MRI demonstrating a right frontal parasagittal tumor that proved to be a meningioma; B) diffusion-weighted sequence axial MRI image three weeks postoperative after a new seizure demonstrating restricted diffusion within the resection cavity that proved to be pus; cultures grew diptheroids and Staph epi; and C) sagittal post-contract CT demonstrating a giant frontal hemorrhage consistent with venous infarction 
Furthermore, hospital records indicate that there were 56 craniotomies performed in the same operating room by the same surgeons following the cessation of use of Kryptonite ${ }^{\circledR}$ from June

2011 to August 2011. There were no post-craniotomy infections during this period. Percentages of post-craniotomy infection in exposed and unexposed groups were $17.8 \%$ and $0.42 \%$, respectively. The relative risk of infection in the exposed group using Kryptonite ${ }^{\circledR}$ versus the unexposed group using plates and screws was 42.1 (95\% CI $=5.10-347.95, P=0.0001)$. The attributable risk in the exposed was 98 percent $(95 \% \mathrm{CI}=0.804-0.997, P=0.0001)$, indicating that 98 percent of the risk of infection in those with bone cement was attributable to the use of bone cement.

\section{Discussion}

In this single-centered retrospective review, Kryptonite ${ }^{\circledR}$ was associated with an increased risk of post-surgical cranial infections relative to plates and screws. There are a number of potential explanations for the increased risk of post-craniotomy infections in surgeries that used Kryptonite ${ }^{\circledR}$. The fact that the Kryptonite ${ }^{\circledR}$ used during the five infected surgeries came from different lot numbers helps to exclude the possibility that one contaminated batch of Kryptonite ${ }^{\circledR}$ might have been responsible, as does the presence of a variety of microorganisms. It is possible that the length of time required for the cement to harden due to its multiple stages of polymerization creates a window of opportunity for incidental contamination from pathogens. The fact that skin flora were responsible for at least four of the infections suggests that the contamination occurs during this time when the scalp is open with the bone exposed, which is a large deviation from what is done with plate and screw fixation.

Furthermore, the normally indolent bacteria, diptheroids and $P$. acnes, rarely cause clinically significant infections without the presence of a foreign body [3].

Substantially after data collection, in March of 2012, the Doctor's Research Group, Inc. released a voluntary recall of Kryptonite ${ }^{\circledR}$ bone cement with expiration dates between January 2010 and April 2012 [4]. The company stated that the characteristcs of the strength and stiffness of cement at body temperature were different from the desired specifications. Though the recall noted complaints of product malleability, there have been no previous reports of adverse events as a result [4].

The principal study limitation is the retrospective design. We also were unable to control for patient factors, such as immunocompromised status, steroid use, and diabetes mellitus, as data was largely unavailable due to the varied patient demographics and incomplete reporting. Suggestions for improvement of the product include incorporating an antibiotic into the composition of the cement so as to prevent implantation of opportunistic pathogens into the drying cement. Over the past three decades, many orthopaedic studies have been conducted to assess the use of antibiotic impregnation and antibiotic releasing techniques for prevention of biofilm formation, yielding varied results which demonstrate the pros and cons of such a technique. While antibiotics have been shown to reduce infection in procedures, such as arthroplasty, there is also the concern of patient allergic reactions and the development of bacterial resistance [5-7]. Some studies showed no difference in infection rate using plain cement versus antibiotic-impregnated cement [6]; yet, there are many potential confounders owing to the broad range of pathogens and the use of appropriate antibiotic. Furthermore, while there is no literature to-date on the topic, another suggestion would be to decrease the polymerization and hardening time of the mixture, so as to prevent contamination of the site in the period postoperatively while the cement is hardening. Other recommendations include that patients wash their hair before surgery, and that postoperative prophylactic systemic antibiotics be given to patients who have had surgery with Kryptonite ${ }^{\circledR}$; however, the latter would not necessarily guard against long-term biofilm formation. 


\section{Conclusions}

In conclusion, considering the potential severity and morbidity associated with cranial infections and that the results of this study indicate an increased risk, it is recommended that Kryptonite ${ }^{\circledR}$ or other similar products not be used and, if so, only with caution, particularly in immunocompromised patients or in patients having re-operation on a previously infected site. However, these data should be investigated with larger, prospective multicenter studies. Despite the findings presented here, there is potential for this or a similar product, as it has demonstrated unique capabilities for skull closure not offered by plates and screws with nice cosmetic results. Thus, we suspect we may see such a product again for use in cranial flap closure, as long as the necessary steps have been taken to optimize its efficacy and safety profile.

\section{Additional Information}

\section{Disclosures}

Human subjects: Consent was obtained by all participants in this study. Inova Human Research Protection Program (formerly IRB) issued approval 12-1063. Animal subjects: All authors have confirmed that this study did not involve animal subjects or tissue. Conflicts of interest: In compliance with the ICMJE uniform disclosure form, all authors declare the following: Payment/services info: All authors have declared that no financial support was received from any organization for the submitted work. Financial relationships: All authors have declared that they have no financial relationships at present or within the previous three years with any organizations that might have an interest in the submitted work. Other relationships: All authors have declared that there are no other relationships or activities that could appear to have influenced the submitted work.

\section{Acknowledgements}

Special thanks to: Dr. Ronan Conroy, DSc, Royal College of Surgeons, Dublin, Ireland and Cherry Herman, RN, Inova Fairfax Hospital, Falls Church, Virginia.

\section{References}

1. McLachlin SD1, Al Saleh K, Gurr KR, Bailey S, Bailey CS, Dunning CE: Comparative assessment of sacral screw loosening augmented with PMMA versus a calcium triglyceride bone cement. Spine (Phila Pa 1976). 2011, 36:E699-704. 10.1097/BRS.0b013e3181fb73ea

2. Levitt MR, Gabikian P, Pottinger PS, Silbergeld DL: Propionibacterium acnes osteomyelitis occurring 23 years after craniotomy: Case report and review of literature. Neurosurg. 2011, 69:E773-9. 10.1227/NEU.0b013e31821964ba

3. Bourne RB: Prophylactic use of antibiotic bone cement: An emerging standard--in the affirmative. J Arthroplasty. 2004, 19:69-72.

4. Gandhi R, Razak F, Pathy R, Davey JR, Syed K, Mahomed NN: Antibiotic bone cement and the incidence of deep infection after total knee arthroplasty. J Arthroplasty. 2009, 24:1015-8. 10.1016/j.arth.2008.08.004

5. Hendriks JG, van Horn JR, van der Mei HC, Busscher HJ: Backgrounds of antibiotic-loaded bone cement and prosthesis-related infection. Biomaterials. 2004, 25:545-56. 